\title{
Genetic modelling in database marketing: The GenIQ Model
}

Received: 8th January, 2004

\author{
Bruce Ratner \\ is a recognised authority in analysis and modelling in the DM space (direct/database marketing, customer relationship \\ management and knowledge discovery/data mining). Dr Ratner is President of DM STAT-1 CONSULTING, which specialises in \\ statistical methods, knowledge discovery and data mining tools. He has applied his expertise in the areas of banking, \\ insurance, finance, retail, telecommunications, healthcare, pharmaceuticals, publication and circulation, mass and direct \\ advertising, catalogue marketing, e-commerce, web-mining, B2B and CRM business. Bruce's latest book Statistical Modelling \\ and Analysis for Database Marketing: Effective Techniques for Mining Big Data is a best seller within its content domain, \\ based on Amazon's sales rank since its publication in June 2003. He is also the co-author of The New Direct Marketing, a \\ text widely studied by direct marketing practitioners, as well as being the author of DM STAT-1 Newsletter, \\ (www.dmstat1.com) the only online information exchange about quantitative methods in the DM space.
}

\begin{abstract}
Using a variety of techniques, data analysts in database marketing aim to build models that maximise expected response and profit from solicitations. Standard techniques include the statistical methods of classical discriminant analysis, as well as logistic and ordinary regression. A recent addition to the data analysis arsenal is the machine learning (ML) method of neural networks. The GenlQ model is a hybrid ML-statistics method that is presented in full detail in this paper.

First, a background on the concept of optimisation will be helpful, since optimisation techniques provide the estimation of all models. Genetic modelling is the 'engine' for the GenlQ model, and is discussed next as an ML optimisation approach. Since the objectives of database marketing are to maximise expected response and profit from solicitations, the author will demonstrate how the GenIQ model serves to meet those objectives. Actual case studies will further explicate the potential of the GenIQ model.
\end{abstract}

Dr Bruce Ratner President, DM STAT-1 Consulting 574 Flanders Drive, North Woodmere, NY 11581, USA.

Tel: +1 516791 3544; Fax: +1516 7915075 e-mail: br@dmstat1.com

\section{WHAT IS OPTIMISATION?}

Whether in business or in model building, optimisation is central to the decision-making process. In both theory and method, optimisation involves selecting the best, or most favourable condition within a given environment. To distinguish among available choices, an objective (or fitness function) must be determined. Whichever choice corresponds to the extreme value ${ }^{1}$ of the objective function is the best alternative and thus the solution to the problem.

Modelling techniques are developed to find a specific solution to a problem. In database marketing, one such problem is to predict sales. The least-squares regression technique is a model formulated to address sales predictions. The regression problem is formulated in terms of finding the regression equation such that the prediction errors (the difference between actual and predicted sales) are small. ${ }^{2}$ The objective function is the prediction error, making the best equation the one that minimises that prediction error. Calculus-based methods are used to estimate the best regression equation.

As discussed later in the paper, each modelling method addresses its own decision problem. The GenIQ model 
addresses a problem specific to database marketing, and uses genetic modelling as the optimisation technique for its solution.

\section{WHAT IS GENETIC MODELLING}

Just as Darwin's principle of the survival of the fittest explains tendencies in human biology, database marketers can use the same principle to predict the best current solution to an optimisation problem. ${ }^{3}$ Each genetic model has an associated fitness value that indicates how well the model solves, or 'fits' the problem. A model with a high fitness value solves the problem better than a model with a lower fitness value - and survives and reproduces at a high rate. Models that are less fit survive and reproduce, at a lower rate if at all.

If two models are effective in solving a problem, then some of their parts undoubtedly contain some valuable genetic material. Recombining the parts of highly fit parent models can sometimes produce offspring models that are a better fit for solving the problem than either parent. Offspring models then become the parents of the next generation, repeating the recombination process. After many generations, an evolved model is declared the best-so-far solution of the problem.

Genetic modelling ${ }^{4}$ consists of the following steps: ${ }^{5}$

1 Definition of the fitness function. The fitness function allows for identifying good or bad models, after which refinements are made with the goal of producing the best model.

2 Selection of a set of functions (eg the set of arithmetic operators - addition, subtraction, multiplication, division $\log$ and exponential) and variables (predictors $\mathrm{X}_{1}, \mathrm{X}_{2}, \ldots, \mathrm{X}_{\mathrm{n}}$ and numerical constants) believed to be related to the problem at hand (the dependent variable $Y) .^{6}$ An initial population of random models must be generated using the preselected set of functions and variables.

3 Calculation of the fitness of each model in the population by applying the model to a training set, a sample of individuals along with their values on the predictor variables $\mathrm{X}_{1}, \mathrm{X}_{2}, \ldots$, $\mathrm{X}_{\mathrm{n}}$ and the dependent variable $Y$.

Thus, every model has a fitness value reflecting how well it solves the problem.

4 Creation of a new population of models by applying the following three operations. (The operations are applied to models in the current population selected with a probability based on fitness - ie the fitter the model, the more likely the model is to be selected.)

- Reproduction: copying an existing model to the new population.

- Crossover: creation of two offspring models for the new population by genetically recombining randomly chosen parts of two existing parent models.

- Mutation: introduction of random changes in some models.

The model with the highest fitness value produced in a generation is declared the best-of-generation model, which may be the solution, or an approximate solution, to the problem.

\section{GENETIC MODELLING: AN ILLUSTRATION}

In the proccess of building a response model, the best model is designated as the one with the highest $R^{2}$ value. Thus, the fitness function is the formula for $R^{2}$ (Analytical note: the $R^{2}$ measure is used for illustration purposes only.) 


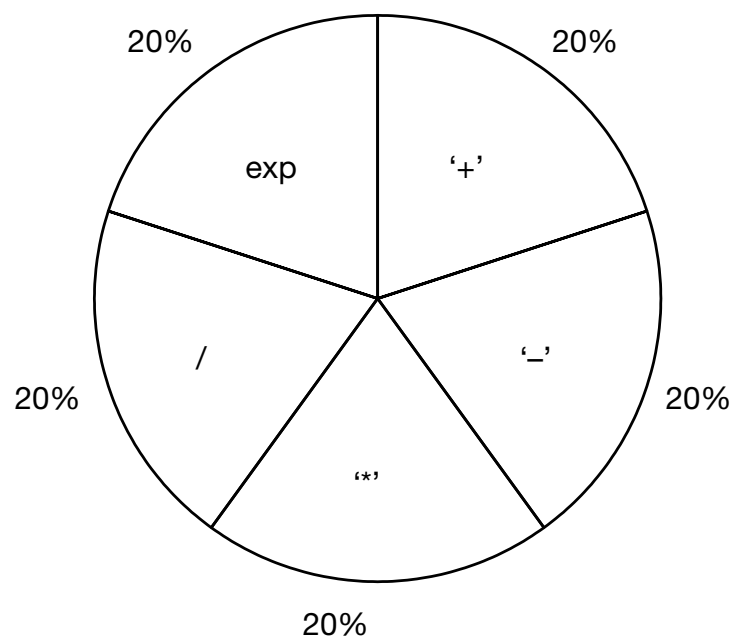

Figure 1: Unbiased function roulette wheel

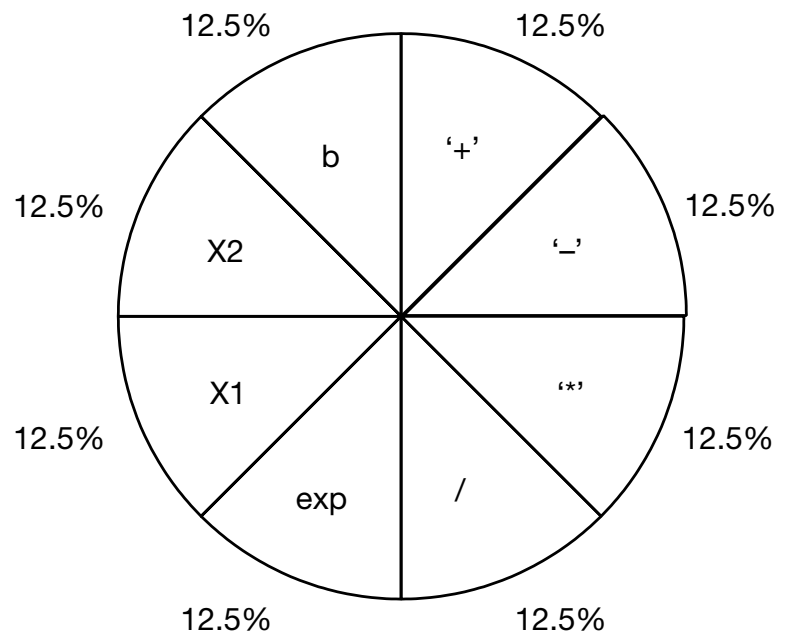

Figure 2: Unbiased function-variable roulette wheel

The modeller has to decide on functions and variables that are related to the problem at hand (eg predicting response). Unless there is theoretical justification - empirical experience usually based on trial and error, provides the guidance for the functions and the variables.

Two variables, $\mathrm{X}_{1}$ and $\mathrm{X}_{2}$, are used as predictors of response. Thus, the variable set contains $\mathrm{X}_{1}$ and $\mathrm{X}_{2}$. Then the numerical constant ' $\mathrm{b}$ ' is added to the variable set based on prior experience. The function set is defined as containing the four arithmetic operations and the exponential function (exp), also based on prior experience.

Generating the initial population is done with an unbiased function roulette wheel, (see Figure 1), and an unbiased function-variable roulette wheel, (see Figure 2). The slices of the function wheel are of equal size, namely, 20 per cent. The slices of the function-variable wheel are also of equal size, namely, 12.5 per cent. Note that the division symbol ' $/$ ' is used to denote a 'protected' division. This 


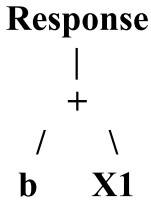

Model 1: Response $=\mathbf{b}+\mathbf{X 1}$

Figure 3: Random model 1

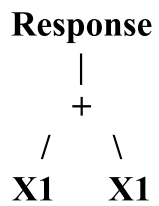

Model 2: Response $=\mathbf{X 1}+\mathbf{X 1}$

Figure 4: Random model 2

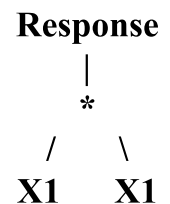

Model 3: Response $=\mathbf{X 1} * \mathbf{X 1}$

Figure 5: Random model 3

means that division by zero is set to the value 1 .

The function wheel is spun to generate the first random model. The wheel's pointer lands on slice ' + '. Next, the function-variable wheel is spun; the pointer lands on slice $\mathrm{X}_{1}$. On the next two spins of the function-variable wheel, the pointer lands on slices ' + ' and ' $b$ ', successively. One can stop evolving the model at this point. The resultant random model 1 is depicted, in Figure 3, as a rooted point-label tree.

To generate the second random model (see Figure 4), the function wheel is spun once and then the function-variable wheel is spun twice. The pointer lands on slices ' + ', $\mathrm{X}_{1}$ and $\mathrm{X}_{1}$, successively. Three more random models, models 3,4 and 5, are generated in a similar way (see

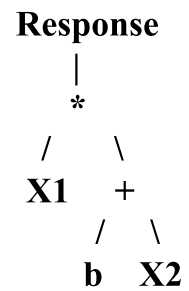

Model 4: Response $=X 1 *(b+X 2)$

Figure 6: Random model 4

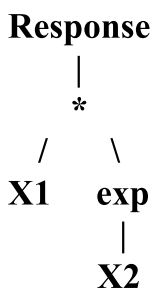

Model 5: Response $=X 1 * \exp (X 2)$

Figure 7: Random model 5

Figures 5, 6 and 7.) Thus, the initial population of five random models has been generated (population size is five).

Each of the five models in the population is assigned a fitness value (see Table 1) indicating how well it solves the problem of predicting response.

Because $R^{2}$ is being used as the fitness function, each model can be applied to a training dataset and its $R^{2}$ value calculated. Model 1 produces the highest $R^{2}$ value, 0.52 , and model 5 produces the lowest $R^{2}$ value, 0.05 .

Fitness for the population itself can be calculated. The total fitness of the population is the sum of the fitness values among all models in the population. Here, the population total fitness is 1.53 (see Table 1).

\section{Reproduction}

After the initial population is generated, the operation of reproduction takes place. Reproduction is the process by which models are duplicated or copied based on 
Table 1: Initial population

\begin{tabular}{lll}
\hline & Fitness value $\left(R^{2}\right)$ & PTF (fitness/total) \\
\hline Model 1 & 0.52 & 0.34 \\
Model 2 & 0.41 & 0.28 \\
Model 3 & 0.38 & 0.25 \\
Model 4 & 0.17 & 0.11 \\
Model 5 & 0.05 & 0.03 \\
Population total fitness & 1.53 & 1 \\
\hline
\end{tabular}

PTF, proportional to fitness

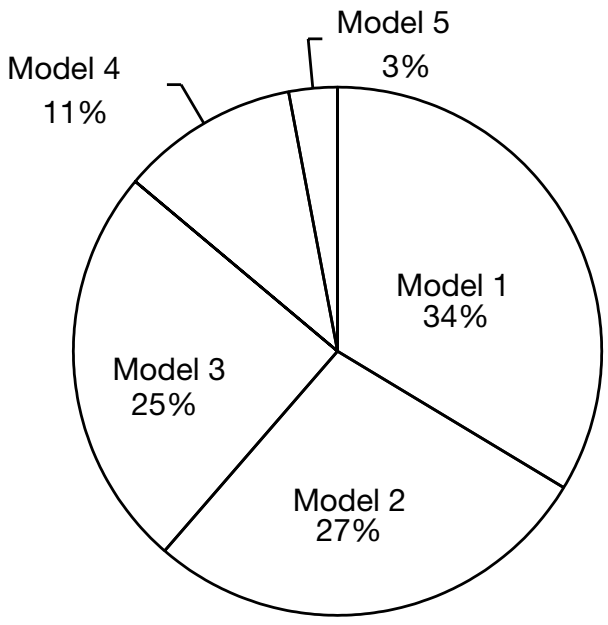

Figure 8: Biased model roulette wheel

selection proportional to fitness (PTF). PTF is defined as model fitness value divided by population total fitness (see Table 1). For example, model 1 has a PTF value of $0.34(=0.52 / 1.53)$.

Reproduction PTF means that a model with a high PTF value has a high probability of being selected for inclusion in the next generation. The reproduction operator is implemented with a biased model roulette wheel (see Figure 8), where the slices are sized according to PTF values.

The operation of reproduction proceeds as follows. The spin of the biased model roulette wheel determines which and how many models are copied. The model selected by the pointer is copied without alteration and put into the next generation. Spinning the wheel in Figure 8, say 100 times, produces, on average, the following selection: 34 copies of model 1, 27 copies of model 2, 25 copies of model 3, 11 copies of model 4 and three copies of model 5 .

\section{Crossover}

The crossover (sexual recombination) operation is performed on two parent models by genetically recombining randomly chosen parts of the two existing parent models; the expectation being that the offspring models are more fit than either parent model.

The crossover operation works with selection PTF. An illustration makes this operation easy to understand. Consider the two parent models in Figures 9 and 10. The operation begins by randomly 


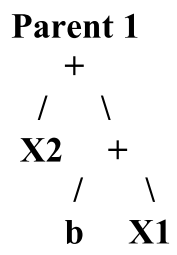

Figure 9: Parent 1

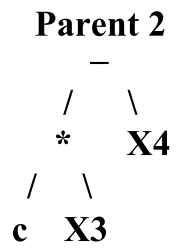

Figure 10: Parent 2

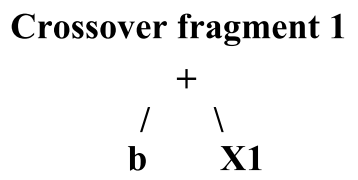

Figure 11: Crossover fragment 1

selecting an internal point (a function) in the tree for the crossover site. Say, the crossover sites are the lower ' + ' and ' $\star$ ' for parents 1 and 2, respectively.

The crossover fragment for a parent is the subtree that has at its root the crossover site function. Crossover fragments for parents 1 and 2 are shown in Figures 11 and 12, respectively. Offspring 1 (see Figure 13) from parent 1 is produced by deleting the crossover fragment of parent 1 and then inserting the crossover fragment of parent 2 at the crossover point of the parent 1 . Offspring 2 (see Figure 14) is produced in a similar way.

\section{Mutation}

The mutation operation begins by selecting a point at random within a tree. This mutation point can be an internal

\section{Crossover fragment 2}

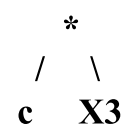

Figure 12: Crossover fragment 2

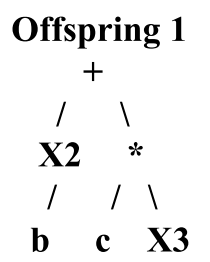

Figure 13: Offspring 1

\section{Offspring 2}

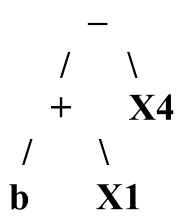

Figure 14: Offspring 2

point (a function) or an external or terminal point (a variable or numerical constant). The mutation operation either replaces a randomly generated function with another function (from the function set previously defined), or inverts ${ }^{8}$ the terminals of the subtree whose root is the randomly selected internal point. ${ }^{8}$

For example, model I, in Figure 15 is mutated by replacing the function '- ' with ' + ', resulting in mutated model I.1 (see Figure 16). Model I is also mutated by inverting the terminal points, $\mathrm{c}$ and X3, resulting in mutated Model I.2 (see Figure 17).

\section{PARAMETERS FOR CONTROLLING A GENETIC MODEL RUN}

There are several control parameters that need to be set before evolving a genetic model. 


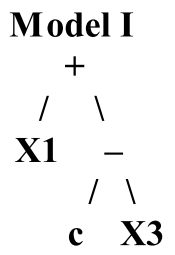

Figure 15: Model I for imputation

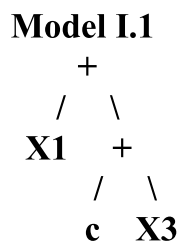

Figure 16: Mutated model I.1

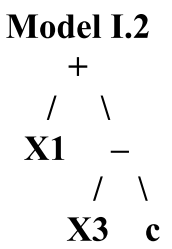

Figure 17: Mutated model I.2

1. Population size, or the number of models randomly generated and subsequently evolved.

2. The maximum number of generations to be run.

3. Reproduction probability, or the percentage of the population which is copied. If population size is 100 and reproduction probability is 10 per cent, then 10 models from each generation are selected (with reselection allowed) for reproduction. Selection is based on PTF.

4. Crossover probability, or the percentage of the population which is used for crossover. If population size is 100 and crossover probability is 80 per cent, then 80 models from each generation are selected (with reselection allowed) for crossover. Selection is based on PTF. Models are paired at random.
5. Mutation probability, or the percentage of the population which is used for mutation. If population size is 100 and mutation probability is 10 per cent, then 10 models from each generation are selected (with reselection allowed) for mutation. Selection is based on PTF

6. Termination criterion, or the single model with the largest fitness value over all generations - the so-called best-so-far model - is declared the result of a run.

\section{GENETIC MODELLING: STRENGTHS AND LIMITATIONS}

Genetic modelling has strengths and limitations, like any methodology.

Perhaps the most important strength of genetic modelling is that it is a workable alternative to traditional methods, which are highly parametric with sample size restrictions. Traditional methods have algorithms depending on smooth, unconstrained functions with the existence of derivatives (well-defined slope values). In practice, the functions (response surfaces) are noisy, multimodal and frequently discontinuous. In contrast, genetic models are robust, assumption-free, non-parametric models, which perform well on large and small samples. The only requirement is a fitness function, which can be designed to insure that the genetic model does not perform worse than any other method.

Genetic modelling has shown itself to be effective for solving large optimisation problems and searching very large datasets. In addition, genetic modelling can be used to learn complex relationships, making it a viable data mining tool for rooting out valuable pieces of information.

A potential limitation of genetic modelling is in the setting of the genetic modelling parameters: population size and 
reproduction, crossover and mutation probabilities. The parameter settings are, in part, data- and problem-dependent, thus proper settings require experimentation. Fortunately, new theories and empirical studies are continually providing rules-of-thumb for these settings as application areas broaden. These guidelines make genetic modelling an accessible approach to analysts not formally trained in this technique. Even with the 'correct' parameter settings, genetic models do not guarantee the optimal (best) solution. Further, genetic models are only as good as the definition of the fitness function. Precisely defining the fitness function, sometimes requires expert experimentation.

\section{GOALS OF MODELLING IN DATABASE MARKETING}

Database marketers typically attempt to improve the effectiveness of their solicitations by targeting their best customers or prospects. They use a model to identify individuals who are likely to respond to or generate profit ${ }^{9}$ from a solicitation. The model provides, for each individual, estimates of probability of response and estimates of contribution-to-profit. Although the precision of these estimates is important, the model's performance is measured at an aggregated level as reported in a decile analysis.

Database marketers have defined the 'cum lift', which is found in the decile analysis, as the relevant measure of model performance (see Table 2). Based on the model's selection of individuals, database marketers create a solicitation list to obtain an advantage over a random selection of individuals. The cum response lift is an index of how many more responses are expected with a selection based on a model over the expected responses with a random selection (without a model). Similarly, the cum profit lift is an index of how much more profit is expected with a selection based on a model over the profit expected with a random selection (without a model).

It should be clear that a model that produces a decile analysis with more responses or profit in the upper (top, 2, 3 or 4) deciles is a better model than a model with fewer responses or less profit in the upper deciles. This concept is the motivation for the GenIQ Model.

(Implementation of the GenIQ theory is available in the GenIQ Software discussed in the Appendix.)

\section{THE GenIQ RESPONSE MODEL}

The GenIQ approach to modelling is to specifically address the objectives concerning database marketers, namely, maximising response and profit from solicitations. The GenIQ Model uses the genetic methodology to explicitly optimise the desired criterion: maximise the upper deciles. Consequently, the GenIQ Model allows data analysts to build response and profit models in ways that are not possible with current methods.

The GenIQ Response Model is theoretically superior - with respect to maximising the upper deciles - to a response model built with alternative response techniques because of the explicit nature of the fitness function. The actual formulation of the fitness function is beyond the scope of this paper, but suffice to say, the fitness function seeks to fill the upper deciles with as many responses as possible.

Alternative response techniques, such as discriminant analysis, logistic regression and artificial neural networks, only implicitly maximise the desired criterion. Their optimisation criterion (fitness function) serves as a surrogate for the desired criterion. These include: 
- Discriminant analysis (DA), with the assumption of bell-shaped data, is defined to explicitly maximise the ratio of between-group sum-ofsquares to within-group sum-of-squares.

- The logistic regression model (LRM), with the two assumptions of independence of responses and an S-shape relationship between predictors and response, is defined to maximise the logistic likelihood function.

- Artificial neural network (ANN), a non-parametric method, is typically defined to explicitly minimise meansquared error (MSE).

\section{THE GenIQ PROFIT MODEL}

The GenIQ Profit Model is theoretically superior - with respect to maximising the upper deciles - to the ordinary least-squares (OLS) regression and ANN. The GenIQ Profit Model uses the genetic methodology with a fitness function that explicitly addresses the desired modelling criterion. This fitness function is defined to fill the upper deciles with as much profit as possible. The fitness function for the OLS and ANN models minimises MSE, which serves as a surrogate for the desired criterion.

OLS regression has another weakness in database marketing applications. A key assumption of the regression technique is that the dependent variable data must follow a bell-shaped curve. If the assumption is violated, the resultant model may not be accurate and reliable. Unfortunately, profit data are not bell-shaped. For example, a 2 per cent response rate yields 98 per cent non-responders with profit values of zero dollars or some nominal cost associated with non-response. Data with a concentration of 98 per cent of a single value cannot be spread out to form a bell-shape distribution.

There is yet another data issue when using OLS with database marketing data. Lifetime value (LTV) is an important database marketing performance measure. LTV is typically positively skewed. The $\log$ is the appropriate transformation to reshape positively skewed data into a normal curve. Using the log of LTV as the dependent variable in OLS regression, however, does not guarantee that other OLS assumptions are not violated. ${ }^{10}$ Accordingly, attempts at modelling profit with ordinary regression is questionable and/or difficult.

The GenIQ Response and Profit Models have no restriction on the dependent variable. They can be accurately and reliably estimated with dependent variables of any shape. ${ }^{11}$ This is because the GenIQ estimation is based on the genetic methodolgy, which is inherently non-parametric and assumption-free.

In fact, due to their non-parametric and assumption-free estimation, the GenIQ Models place no restriction on the inter-relationship among the independent variables. The GenIQ Models are virtually unaffected by any degree of correlation among the independent variables. By contrast, OLS and ANN, as well as DA and LRM, can tolerate only a 'moderate' degree of correlation among the independent variables to ensure a stable calculation of their models.

Moreover, the GenIQ Models have no restriction on sample size. They can be built on small samples as well as large samples. OLS and DA and, to a lesser extent ANN and $\mathrm{LRM}^{12}$ models require at least a 'moderate' size sample. ${ }^{13}$

\section{CASE STUDY - RESPONSE MODEL}

Cataloguer $\mathrm{ABC}$ requires a response model based on a recent direct mail 
Table 2: Response decile analysis

\begin{tabular}{lcclll}
\hline Decile & $\begin{array}{l}\text { Number of } \\
\text { individuals }\end{array}$ & $\begin{array}{l}\text { Number of } \\
\text { responses }\end{array}$ & $\begin{array}{l}\text { Decile } \\
\text { response } \\
\text { rate (\%) }\end{array}$ & $\begin{array}{l}\text { Cumulative } \\
\text { response } \\
\text { rate (\%) }\end{array}$ & $\begin{array}{l}\text { Cumulative } \\
\text { lift }\end{array}$ \\
\hline Top & 7,410 & 911 & 12.30 & 12.30 & 294 \\
2 & 7,410 & 544 & 7.30 & 9.80 & 235 \\
3 & 7,410 & 437 & 5.90 & 8.50 & 203 \\
4 & 7,410 & 322 & 4.30 & 7.50 & 178 \\
5 & 7,410 & 258 & 3.50 & 6.70 & 159 \\
6 & 7,410 & 188 & 2.50 & 6.00 & 143 \\
7 & 7,410 & 130 & 1.80 & 5.40 & 129 \\
8 & 7,410 & 163 & 2.20 & 5.00 & 119 \\
9 & 7,410 & 124 & 1.70 & 4.60 & 100 \\
Bottom & 7,410 & 24 & 0.30 & 4.20 & \\
Total & 74,100 & 3,101 & 4.20 & & \\
\hline
\end{tabular}

Table 3: Logistic regression model response decile analysis

\begin{tabular}{lcclll}
\hline Decile & $\begin{array}{l}\text { Number of } \\
\text { individuals }\end{array}$ & $\begin{array}{l}\text { Number of } \\
\text { responses }\end{array}$ & $\begin{array}{l}\text { Decile } \\
\text { response } \\
\text { rate (\%) }\end{array}$ & $\begin{array}{l}\text { Cumulative } \\
\text { response } \\
\text { rate (\%) }\end{array}$ & $\begin{array}{l}\text { Cumulative } \\
\text { response lift }\end{array}$ \\
\hline Top & 1,740 & 38 & 2.20 & 2.18 & 264 \\
2 & 1,740 & 12 & 0.70 & 1.44 & 174 \\
3 & 1,740 & 18 & 1.00 & 1.30 & 157 \\
4 & 1,740 & 12 & 0.70 & 1.15 & 139 \\
5 & 1,740 & 16 & 0.90 & 1.10 & 133 \\
6 & 1,740 & 20 & 1.10 & 1.11 & 134 \\
7 & 1,740 & 8 & 0.50 & 1.02 & 123 \\
8 & 1,740 & 10 & 0.60 & 0.96 & 116 \\
9 & 1,740 & 6 & 0.30 & 0.89 & 108 \\
Bottom & 1,740 & 4 & 0.20 & 0.83 & \\
Total & 17,400 & 144 & 0.83 & & 100 \\
\hline
\end{tabular}

campaign, which produced a 0.83 per cent response rate. ABC's consultant built an LRM using three variables:

1 RENT_1 - a composite variable measuring the ranges of rental cost. ${ }^{14}$

2 ACCT_1 - a composite variable measuring the activity of various financial accounts. ${ }^{15}$

3 APP_TOTL - the number of inquiries.

The LRM is defined in Equation (1) as:

$$
\begin{aligned}
& \text { RESPONSE }=-1.9+0.19 \star \\
& \text { APPTOTL }-0.24 \star \\
& \text { RENT_1 }-0.25^{\star} \text { ACCTS_1 }
\end{aligned}
$$

The LRM response decile analysis in

Table 3 shows the performance of the model over chance (ie no model). The decile analysis shows a model with good performance in the upper deciles: Cum lifts for the top, 2, 3, and 4 deciles are 264, 174, 157 and 139, respectively. Note that the model may not be as good as initially believed. There is some degree of unstable performance through the deciles; ie the number of responses does not decrease steadily through the deciles. This unstable performance, which is characterised by 'jumps' in deciles 3, 5,6 , and 8 , is probably due to unknown structure or relationships between the three predictors and responses. The implication is that the LRM assumption of global linearity (ie all predictors are linearly related to response) is not justified. It should be pointed out, however, that only perfect models have 


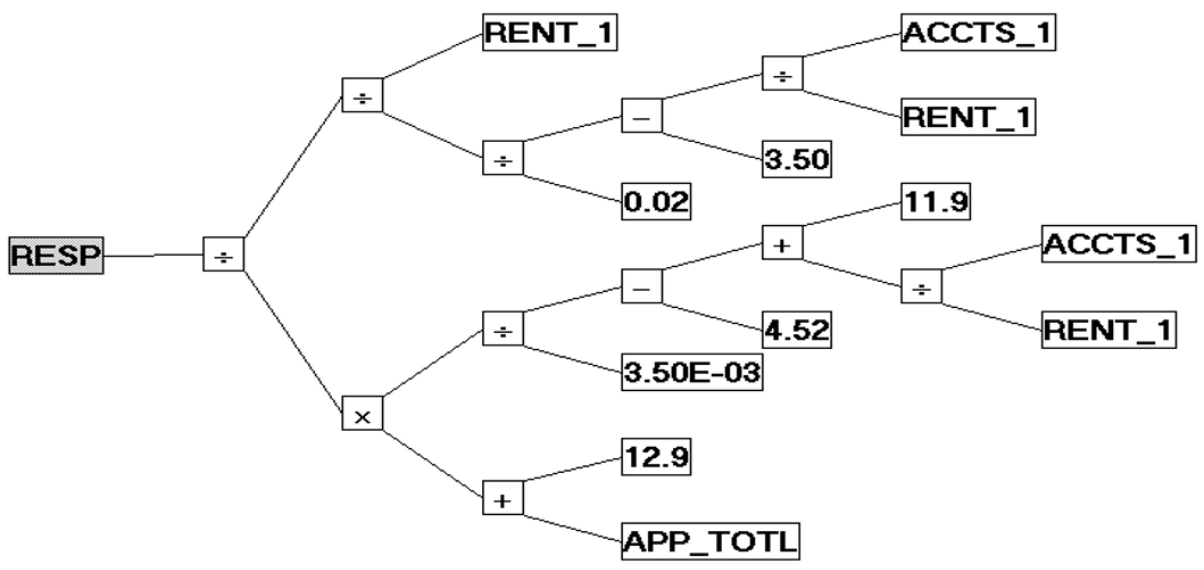

Figure 18: GenIQ response tree

perfect performance through the deciles. Good models have some jumps, albeit minor ones.

A GenIQ Response Model based on the same three variables used in the LRM was built. The GenIQ Response tree is shown in Figure 18. The decile analysis, in Table 4, shows a model with very good performance in the upper deciles: cum lifts for the top, 2, 3, and 4 deciles are 306, 215, 167 and 142. In contrast to the LRM, the GenIQ Model has only two minor jumps - in deciles 5 and 7. The implication is that the genetic methodology has evolved a better model because it has uncovered a non-linear relationship among the predictors and responses.

The GenIQ Response Model is defined in Equation (2) as:

RESPONSE $=$

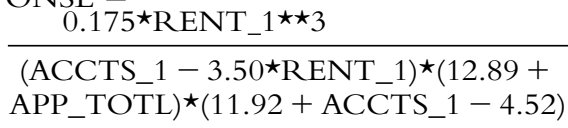

GenIQ does not outperform LRM across all the deciles in Table 5. However, GenIQ yields noticeable cum lift improvements for the important first three decile ranges of 16.0 per cent, 23.8 per cent, and 6.1 per cent, respectively.

\section{CASE STUDY - PROFIT MODEL}

Telecommunications company ATMC seeks to build a zip code-level model to predict usage, TTLDIAL1. The variables used in building an OLS model are:

1 AASSIS_1 - composite of public assistance-related census variables.

2 ANNTS_2 - composite of ancestry census variables.

3 FEMMAL_2 - composite of gender-related variables.

4 FAMINC_1 - a composite variable measuring the ranges of home value. ${ }^{16}$

The OLS profit model is defined in Equation (3) as:

$$
\begin{aligned}
& \text { TTLDIAL1 }=1.5+ \\
& -0.35^{\star} \text { AASSIS_1 }+1.1 \star \text { ANNTS_2 } \\
& +1.4 \star \text { FEMMAL_2 } \\
& +2.8 \star \text { FAMINC_1 }
\end{aligned}
$$

The OLS profit decile analysis in Table 6 shows the performance of the model over chance (ie no model). The analysis shows a model with good performance in the upper deciles: cum lifts for the top, 2, 3, and 4 deciles are 158, 139, 131 and 123 , respectively.

A GenIQ Profit Model was built, based on the same four variables used in the 
Table 4: GenIQ response decile analysis

\begin{tabular}{lcclll}
\hline Decile & $\begin{array}{l}\text { Number of } \\
\text { individuals }\end{array}$ & $\begin{array}{l}\text { Number of } \\
\text { responses }\end{array}$ & $\begin{array}{l}\text { Decile } \\
\text { response } \\
\text { rate (\%) }\end{array}$ & $\begin{array}{l}\text { Cumulative } \\
\text { response } \\
\text { rate (\%) }\end{array}$ & $\begin{array}{l}\text { Cumulative } \\
\text { lift }\end{array}$ \\
\hline Top & 1,740 & 44 & 2.50 & 2.53 & 306 \\
2 & 1,740 & 18 & 1.00 & 1.78 & 215 \\
3 & 1,740 & 10 & 0.60 & 1.38 & 167 \\
4 & 1,740 & 10 & 0.60 & 1.18 & 142 \\
5 & 1,740 & 14 & 0.80 & 1.10 & 133 \\
6 & 1,740 & 10 & 0.60 & 1.02 & 123 \\
7 & 1,740 & 12 & 0.70 & 0.97 & 117 \\
8 & 1,740 & 10 & 0.60 & 0.92 & 105 \\
9 & 1,740 & 8 & 0.50 & 0.87 & 100 \\
Bottom & 1,740 & 8 & 0.50 & 0.83 & \\
Total & 17,400 & 144 & 0.83 & & \\
\hline
\end{tabular}

Table 5: Comparison of logistic regression model (LRM) and GenlQ responses

\begin{tabular}{llll}
\hline Decile & LRM & GenIQ & $\begin{array}{l}\text { GenlQ improvement } \\
\text { over LRM }\end{array}$ \\
\hline Top & 264 & 306 & 16.00 \\
2 & 174 & 215 & 23.80 \\
3 & 157 & 167 & 6.10 \\
4 & 139 & 142 & 2.20 \\
5 & 133 & 133 & -0.20 \\
6 & 134 & 123 & -8.20 \\
7 & 123 & 117 & -4.90 \\
8 & 116 & 111 & -4.60 \\
9 & 108 & 105 & -2.80 \\
Bottom & 100 & 100 & - \\
\hline
\end{tabular}

Table 6: Decile analysis of ordinary least-squares profit model

\begin{tabular}{lccccc}
\hline Decile & $\begin{array}{l}\text { Number of } \\
\text { customers }\end{array}$ & $\begin{array}{l}\text { Total dollar } \\
\text { usage }\end{array}$ & $\begin{array}{l}\text { Average } \\
\text { usage }\end{array}$ & $\begin{array}{l}\text { Cumulative } \\
\text { average } \\
\text { usage }\end{array}$ & $\begin{array}{l}\text { Cumulative } \\
\text { usage }\end{array}$ \\
\hline Top & 1,800 & $\$ 38,379$ & $\$ 21.32$ & $\$ 21.32$ & 158 \\
2 & 1,800 & $\$ 28,787$ & $\$ 15.99$ & $\$ 18.66$ & 139 \\
3 & 1,800 & $\$ 27,852$ & $\$ 15.47$ & $\$ 17.60$ & 131 \\
4 & 1,800 & $\$ 24,199$ & $\$ 13.44$ & $\$ 16.56$ & 123 \\
5 & 1,800 & $\$ 26,115$ & $\$ 14.51$ & $\$ 16.15$ & 120 \\
6 & 1,800 & $\$ 18,347$ & $\$ 10.19$ & $\$ 15.16$ & 113 \\
7 & 1,800 & $\$ 20,145$ & $\$ 11.19$ & $\$ 14.59$ & 108 \\
8 & 1,800 & $\$ 23,627$ & $\$ 13.13$ & $\$ 14.41$ & 107 \\
9 & 1,800 & $\$ 19,525$ & $\$ 10.85$ & $\$ 14.01$ & 104 \\
Bottom & 1,800 & $\$ 15,428$ & $\$ 8.57$ & $\$ 13.47$ & 100 \\
Total & 18,000 & $\$ 242,404$ & $\$ 13.47$ & & \\
\hline
\end{tabular}

OLS model. The GenIQ Profit Tree is shown in Figure 19. The decile analysis in Table 7 shows a model with very good performance in the upper deciles: cum lifts for the top, 2, 3, and 4 deciles are 198, 167, 152 and 140, respectively.

The GenIQ Profit Model is defined in equation (4) as:

$$
\begin{aligned}
& \text { TTLDIAL1 }=+5.95+\text { FAMINC_1 } \\
& +\left((\text { FAMINC_1 }+ \text { AASSIS_1 })^{\star}\right. \\
& (0.68 \star \text { FEMMAL_2 })^{\star}(\text { AASSIS_1 } \\
& -3.485))
\end{aligned}
$$

GenIQ does outperform OLS across all the deciles in Table 8 and yields noticeable cum lift improvements down 
Table 7: Decile analysis of GenIQ Profit Model

\begin{tabular}{lccccc}
\hline Decile & $\begin{array}{l}\text { Number of } \\
\text { customers }\end{array}$ & $\begin{array}{l}\text { Total dollar } \\
\text { usage }\end{array}$ & $\begin{array}{l}\text { Average } \\
\text { usage }\end{array}$ & $\begin{array}{l}\text { Cumulative } \\
\text { average } \\
\text { usage }\end{array}$ & $\begin{array}{l}\text { Cumulative } \\
\text { usage }\end{array}$ \\
\hline Top & 1,800 & $\$ 48,079$ & $\$ 26.71$ & $\$ 26.71$ & 198 \\
2 & 1,800 & $\$ 32,787$ & $\$ 18.22$ & $\$ 22.46$ & 167 \\
3 & 1,800 & $\$ 29,852$ & $\$ 16.58$ & $\$ 20.50$ & 152 \\
4 & 1,800 & $\$ 25,399$ & $\$ 14.11$ & $\$ 18.91$ & 140 \\
5 & 1,800 & $\$ 25,115$ & $\$ 13.95$ & $\$ 17.91$ & 133 \\
6 & 1,800 & $\$ 18,447$ & $\$ 10.25$ & $\$ 16.64$ & 124 \\
7 & 1,800 & $\$ 16,145$ & $\$ 8.97$ & $\$ 15.54$ & 115 \\
8 & 1,800 & $\$ 17,227$ & $\$ 9.57$ & $\$ 14.80$ & 110 \\
9 & 1,800 & $\$ 15,125$ & $\$ 8.40$ & $\$ 14.08$ & 105 \\
Bottom & 1,800 & $\$ 14,228$ & $\$ 7.90$ & $\$ 13.47$ & \\
Total & 18,000 & $\$ 242,404$ & $\$ 13.47$ & & \\
\hline
\end{tabular}

Table 8: Comparison of ordinary least-squares (OLS) and GenIQ Profit Models

\begin{tabular}{llll}
\hline Decile & OLS & GenIQ & $\begin{array}{l}\text { GenIQ improvement } \\
\text { over OLS (\%) }\end{array}$ \\
\hline Top & 158 & 198 & 25.50 \\
2 & 139 & 167 & 20.00 \\
3 & 131 & 152 & 16.20 \\
4 & 123 & 140 & 14.10 \\
5 & 120 & 133 & 10.90 \\
6 & 113 & 124 & 9.30 \\
7 & 108 & 115 & 6.90 \\
8 & 107 & 110 & 2.70 \\
9 & 104 & 105 & 0.60 \\
Bottom & 100 & 100 & - \\
\hline
\end{tabular}

to the seventh decile. Improvements range from 25.5 per cent in the top decile to 6.9 per cent in the seventh decile.

\section{SUMMARY}

All standard statistical modelling techniques involve optimising a fitness function to find a specific solution to a problem. The popular ordinary and logistic regression techniques, which seek accurate prediction and classification, respectively, optimise the fitness function, MSE and the logistic likelihood (LL), respectively. Calculus-based methods are used for the optimisation computations.

This paper presented a new modelling technique, GenIQ Model, which seeks maximum performance (response or profit) from solicitations. The GenIQ
Model optimises the fitness function cum lift. GenIQ's optimisation computations use the genetic methodology, not the usual calculus. A compendious introduction to genetic methodology with an illustration and inspection of its strengths and limitations was also provided.

The GenIQ Model is theoretically superior - with respect to maximising cum lift - to the ordinary and logistic regression models because of its clearly and fully formulated fitness function. The GenIQ fitness function explicitly seeks to fill the upper deciles with as many responses or profit as possible. Standard statistical methods only implicitly maximise the cum lift, as their fitness functions (MSE and LL) serve as surrogate maximising cum lifts.

Lastly, the potential of the new 


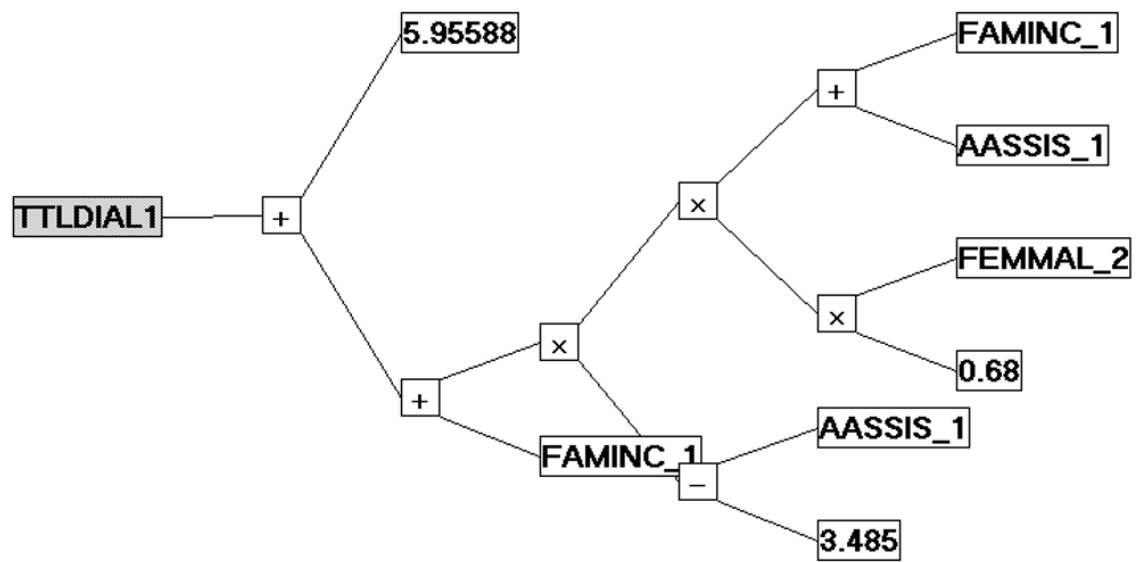

Figure 19: GenlQ Profit Tree

technique was demonstrated using response and profit model illustrations. The GenIQ response illustration yields noticeable cum lift improvements over logistic regression for the important first three decile ranges: 16.0 per cent, 23.8 per cent, and 6.1 per cent, respectively. The GenIQ profit illustration yields noticeable cum lift improvements over ordinary regression down to the seventh deciles ranging from 25.5 per cent in the top deciles to 6.9 per cent in the seventh decile.

\section{APPENDIX - GenIQ SOFTWARE WHO \& WHY}

Dr Bruce Ratner realised several years ago that the standard statistical models of logistic and ordinary regression for binary and continuous target variables, respectively, have two weaknesses:

1. They do not address the DM objective of maximising model lift the customary performance measure used in the DM space.

2. They do not have built-in data mining capability - the intelligence for constructing new variables with incremental predictiveness beyond the original variables.
For these reasons Dr Ratner invented the assumption-free, non-parametric GenIQ Model, which addresses the weaknesses of the statistical DM problem-solution, by using the machine learning (ML) method of genetic programming (GP).

\section{WHAT}

GenIQ software is the PC-based implementation of the GenIQ Model for building:

1. Classification models - the target variable is a binary (eg yes-no response).

2. Prediction models - the target variable is continuous (eg many-valued profit).

GenIQ software is flexible during the data input and output processes it:

1. Imports unlimited records and candidate predictor variables of any type (categorical, ordinal and continuous).

2. Reduces the mandatory exploratory data analysis to checking only for implausible and impossible values due to the consequences of benefits \#1 and \#5 listed below. 
3. Efficiently accommodates the problem of missing data with genetic imputation.

4. Exports effortlessly the GenIQ Model equation into any independent application for error-free scoring of the model to an external file or database.

\section{HOW}

GenIQ Software has only four simple screens:

1. Input file - to indicate the input data location and file type (eg SAS, SPSS, Excel, text and comma delimited).

2. Identify variables - to identify the target and candidate predictor variables.

3. Genetic parameters - to scan the default control settings for the run. Fiddling with the settings is rarely needed, as the GP-approach to maximising model lift is quite resistant.

4. GenIQ Model - to view the model in its signature 'tree' display, not the typical ML indiscernible 'black box.'

\section{BENEFITS}

The following features of the GenIQ Model directly translate into significant value-added benefits of the GenIQ software.

1. The GenIQ Model has maximum model lift, as its best-of-generation solution estimates the rank-order likelihood of response or contribution of profit.

Value-added: greater response/profit leading to increased cost efficiency in DM programs

2. The GenIQ Model is more reliable than the logistic and ordinary regression models because it lets the data suggest the equation form without any assumptions. The regression models require the rigid assumption of the equation form (sum of weighted predictor variables) and a host of other untenable assumptions. Value-added: greater model reliability leading to increased confidence in the predicted DM program results.

3. The GenIQ Model is automatically built in a time frame favourably commensurate with the input file size. Model validation is quickly and easily performed.

Value-added: greater rate per model built leading to increased human captial.

4. The GenIQ Model can serve as a productivity tool for data analysts who build models in the DM space where maximising model lift is the bottom-line objective.

Value-added: greater productive labour leading to increased quantity of models built

5. The GenIQ Model can serve as a specialty tool for experts who want control over the individual processes: selecting among the important original variables, assessing the predictiveness of the newly data-mined variables and developing the best model with maximum model lift.

Value-added: greater 'creativity' afforded leading to increased quality of models built and individuality of human captial.

6. The GenIQ Model provides automatic data mining - an inherent byproduct of the genetic programming methodology. Data analysts can export the genetically constructed variables into an independent model application. Value-added: presents hybrid statistics ML paradigm that yields a utile alternative for DM modelling. ${ }^{17}$

7. The GenIQ Model provides a unique variable selection of important predictor variables, as it provides the ranking of the relationship between 
each predictor variable with the target variable - accounting for the presence of the other predictor variables jointly considered. This is in stark contrast to the statistical correlation coefficient, which provides the ranking of the linear-relationship between each predictor variable with the target variable - without considering the other predictor variables.

Value-added: presents a hybrid statistics-ML paradigm that yields a utile alternative for DM modelling.

\section{DISADVANTAGE}

The GenIQ Model - represented as a tree - is difficult to interpret, in part, because it has no coefficients. Tyros and experienced analysts, when interpreting a model, unwittingly seek the regression coefficients, as they are the means to interpreting the everyday logistic and ordinary regression models.

\section{NON-DISADVANTAGE}

The GenIQ Model - represented as a set of 'branches' - lends itself to interpretation even without coefficients. The branches, which are actually small models that are defined with a few variables, can be explained with an understanding of the content domain of the variables. Viewing the GenIQ Model as such, data analysts and end-users acquire a comfort level for using the model that is otherwise difficult to understand and accept.

\section{References and notes}

1 If the optimisation problem seeks to minimise the objective function, then the extreme value is the smallest; if it seeks to maximise, then the extreme value is the largest.
2 The definition of 'small' (technically called mean squared error) is the average of the squared differences between actual and predicted values.

3 The focus of this chapter is optimisation, but, genetic modelling has been applied to a variety of problems: optimal control; planning; sequence induction; empirical discovery and forecasting; symbolic integration; and discovering mathematical identities.

4 Genetic modelling as described in this chapter is formally known as genetic programming. The author has chosen the term 'modelling' instead of 'programming' because the latter term, which has its roots in computer sciences, does not connote the activity of model building to data analysts with statistics or quantitative backgrounds.

5 Koza, J. (1992) 'Genetic Programming: On the Programming of Computers by Means of Natural Selection', The MIT Press, Cambridge, MA.

6 Effectively, a genetic alphabet has been chosen.

$7 R^{2}$ is not an appropriate fitness function for a $0-1$ dependent variable model. Possibly the likelihood function of the logistic regression model should be used as the fitness measure for the response model, or an example with a continuous (profit) variable and the $R^{2}$ fitness measure. There is more about the appropriate choice of fitness function for the problem at hand in a later section.

8 When a subtree has more than two termini, the termini are randomly permutated.

9 I use the term 'profit' as a stand-in for any measure of an individual's worth, such as, sales per order, lifetime sales, revenue, number of visits, or number of purchase.

10 The error structure of the OLS equation may not necessarily be normally distributed with zero mean and constant variance, in which case the modelling results are questionable, and additional transformations may be needed.

11 The dependent variable can be bell-shaped or skewed, bimodal or multimodal, continuous or discontinuous.

12 There are specialty algorithms for logistic regression with small sample size.

13 Statisticians do not agree on how 'moderate' a moderate sample size is. The author drew a sample of statisticians to determine the size of a moderate sample; the average was 5,000.

14 Four categories of rental cost: less than $\$ 200$ per month, $\$ 200-\$ 300$ per month, $\$ 300-\$ 500$ per month and greater than $\$ 500$ per month.

15 Financial accounts include bank cards, department store cards, installments on loans etc.

16 Seven categories of home value: less than $\$ 100 \mathrm{~m}$, $\$ 100-\$ 200 \mathrm{~m}, \$ 200-\$ 500 \mathrm{~m}, \$ 500-\$ 750 \mathrm{~m}$, greater than $\$ 750 \mathrm{~m}$.

17 See article 'A Hybrid Statistics-Machine Learning Paradigm for DM Modeling' http://www.dmstat1.com/49.html.) 